\title{
Novel genetic changes in Autosomal Dominant, Primary Macronodular Adrenal Hyperplasia associated with hypercortisolism and giant adrenals
}

\begin{abstract}
Munter $\mathbf{G}^{1}$, Beeri $\mathrm{R}^{2}$, Altarescu $\mathrm{G}^{2}$, Weiss $\mathrm{R}^{2}$, Berthon $\mathrm{A}^{3}$, Faucz $\mathrm{FR}^{3}$ and Stratakis $\mathrm{CA}^{3}$
${ }^{1}$ Internal Medicine $\mathrm{C}$ and Endocrine Unit, Shaare Zedek Medical Center affiliated with Faculty of Medicine-Hebrew University, Jerusalem, ${ }^{2}$ Genetics Department, Shaare Zedek Medical Center affiliated with Faculty of Medicine-Hebrew University, Jerusalem, ${ }^{3}$ Section on Endocrinology and Genetics (SEGEN), Program on Developmental Endocrinology and Genetics (PDEGEN), Eunice Kennedy Shriver National Institute of Child Health and Human Development (NICHD), National Institutes of Health (NIH), Bethesda, MD, USA.
\end{abstract}

\section{OBJECTIVES}

- Primary macronodular adrenal hyperplasia (PMAH) is a rare cause of adrenal hypercortisolism.

- A family with Autosomal Dominant PMAH with adrenal hypercortisolism was studied. Aberrant receptors studies were negative.

- ARMC5 has been associated with PMAH and adrenal tumors $(1,2)$.

- cAMP-dependent protein kinase A (PKA) signaling is the major activator of cortisol secretion in the adrenal cortex and mutations in PDE11A4 has been associated with PMAH(3).

- The aim of this study was to reveal the genetic basis of ADPMAH studying PDE11A4 and ARMC5 genes.
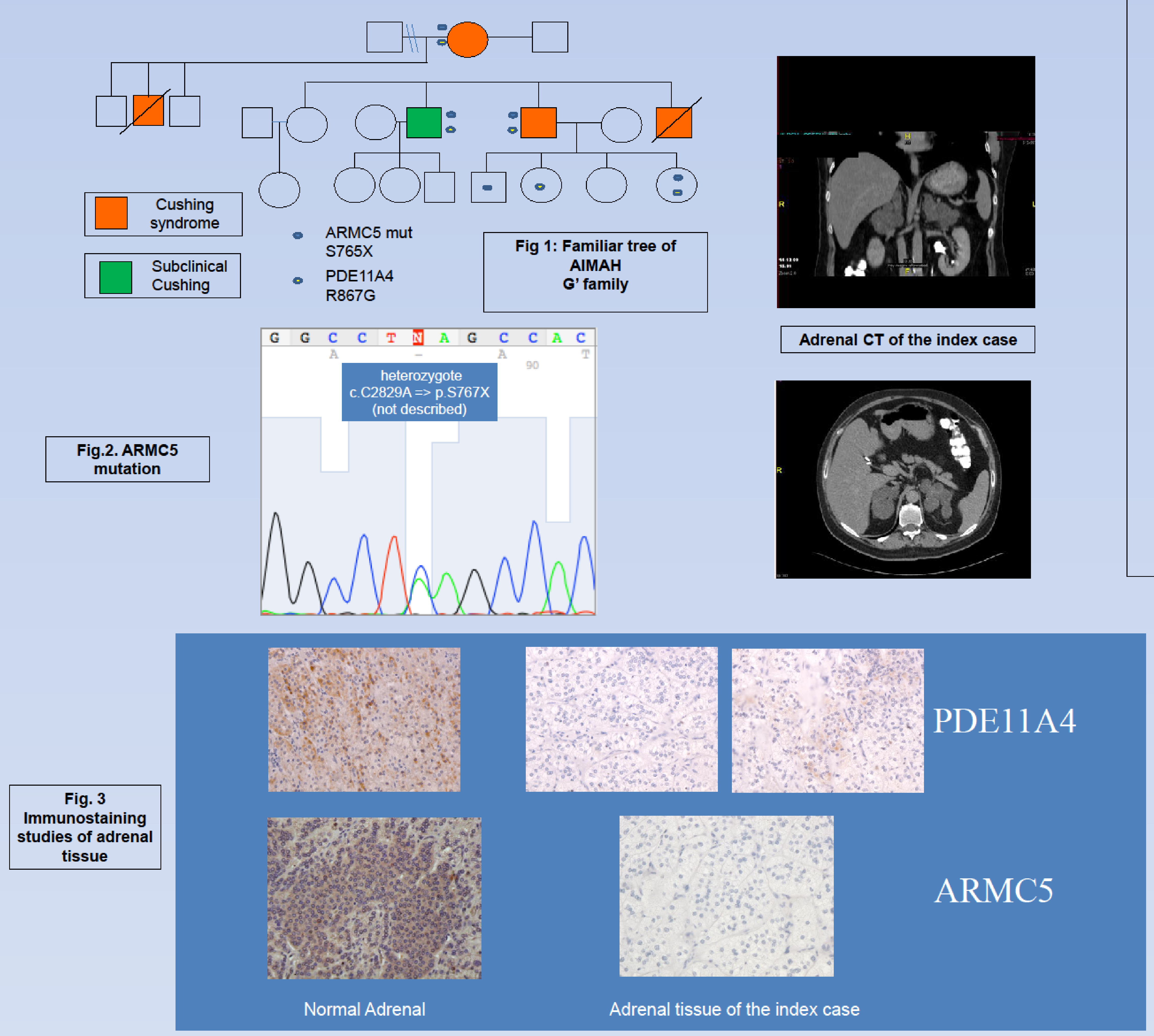

CONCLUSIONS

- A family with ADPMAH causing giant adrenal hyperplasia associated with a novel mutation in ARMC5 in conjunction with PDE11A4 mutation, causing low protein expression is reported.

- Coexistence of PDE11A4 variant in all three affected individuals may indicate a phenotype modifier role.

- Because clinical and biochemical abnormalities appear during adulthood, young phenotypically normal mutation carriers may be at risk of developing clinical disease in the future per MRI.

\section{METHODS}

- Bilateral adrenalectomy was performed. The adrenal weight was 470 gr. Clinical evaluation of the family (Fig 1) was carried out including biochemical, hormonal and imaging studies (CT/MRI).

- DNA was extracted from lymphocytes and sequencing of PDE11A4 and ARMC5 genes was performed in the index case. Screening for the found mutations was done in the family.

- Immunohistochemical studies for PDE11A4 and ARMC5 in the adrenal tissue of the index patient was performed.

- A germline heterozygote rare variant of PDE11A4 R867G (2-3\% frequency in general population) was found(4) as well as a novel germline heterozygote mutation of ARMC5 mutation (Fig 2).

- Adrenal protein expression of PDE11A4 and ARMC5 in the adrenal gland of the index case was very low (Fig 3).

- All the patients with hypercortisolism harbor both ARMC5 and PDE11A4 mutations.

- Young patients with one or both mutations had a normal HPA axis and the adrenal glands had a normal appearance

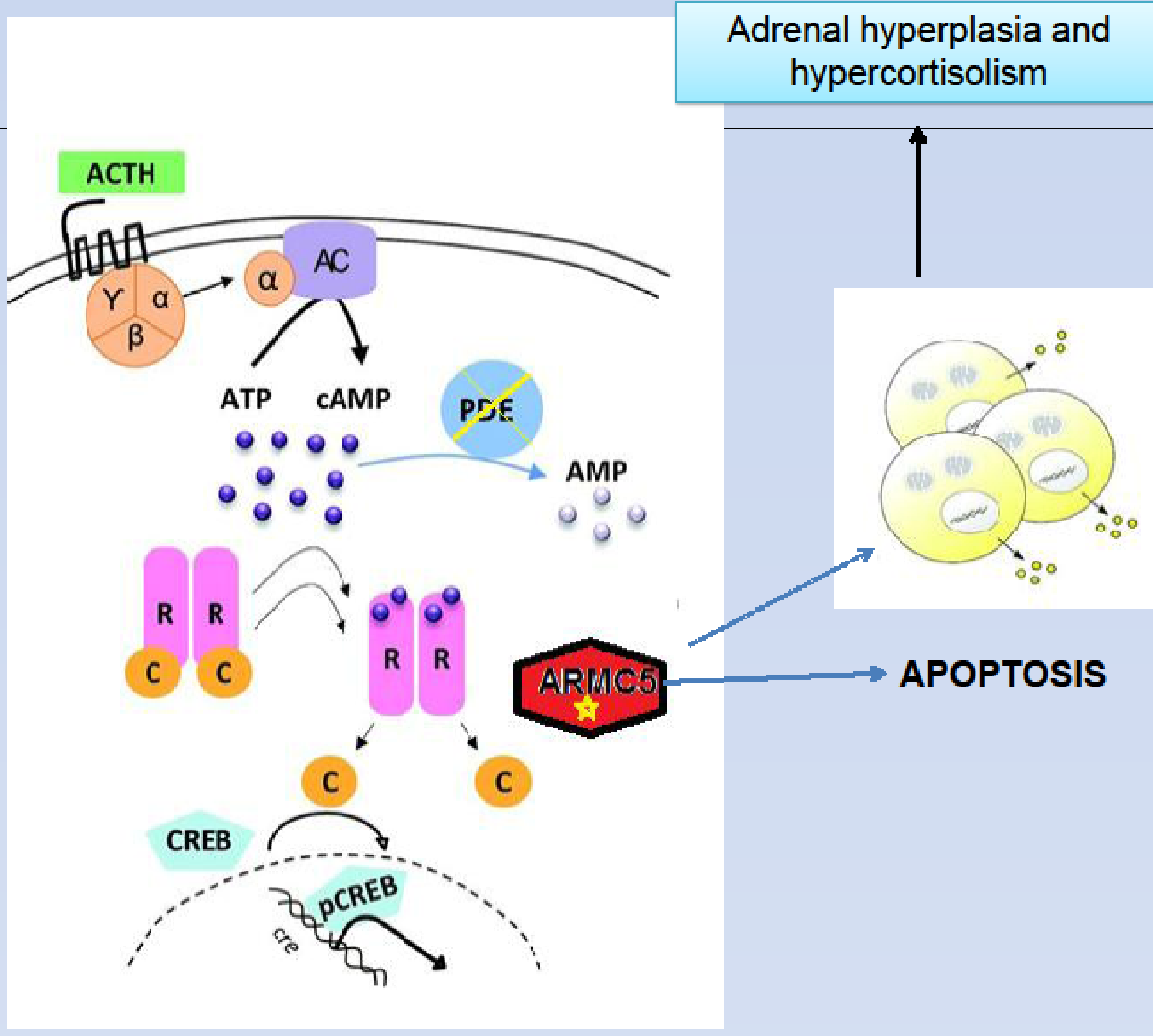

REFERENCES: 